\title{
Short communication: Coliform Petrifilm as an alternative method for detecting total gram-negative bacteria in fluid milk
}

\author{
A. Rojas, S. I. Murphy, () M. Wiedmann, (1) and N. H. Martin* () \\ Milk Quality Improvement Program, Department of Food Science, Cornell University, Ithaca, NY 14853
}

\begin{abstract}
Postpasteurization contamination (PPC) of fluid milk remains a challenge for some dairy processors. Pseudomonas is the most common contaminant of fluid milk after pasteurization, and therefore methods to detect PPC should be inclusive of Pseudomonas and other gram-negative contaminants (e.g., coliform bacteria). Our objective was to compare the ability of $3 \mathrm{M}$ (St. Paul, MN) coliform and Enterobacteriaceae (EB) Petrifilm to detect total gram-negative bacteria with that of the standard method, crystal violet tetrazolium agar. To that end, we evaluated coliform Petrifilm, EB Petrifilm, and crystal violet tetrazolium agar to detect gram-negative bacteria in naturally contaminated samples of fluid milk. A total of 92 observations derived from shelf-life testing of 33 milk samples from 5 different processing facilities were evaluated for (1) presence of coliforms on coliform Petrifilm at both 24 and $48 \mathrm{~h}$ of incubation; (2) presence of any growth, regardless of gas production, on coliform Petrifilm at both 24 and $48 \mathrm{~h}$ of incubation; (3) presence of EB on EB Petrifilm at both 24 and $48 \mathrm{~h}$ of incubation; (4) presence of any growth, regardless of gas or acid production, on EB Petrifilm at both 24 and $48 \mathrm{~h}$ of incubation; and (5) presence of gram-negative bacteria on crystal violet tetrazolium agar after $48 \mathrm{~h}$ of incubation. Sensitivity and specificity analysis of results indicated that compared with the standard method (i.e., crystal violet tetrazolium agar), the method that performed the best, based on balanced accuracy (i.e., the average of sensitivity and specificity), was coliform Petrifilm evaluated for the presence of any growth after $48 \mathrm{~h}$ of incubation (sensitivity $=0.787$; specificity $=0.839)$. This method can be easily adopted by the dairy industry as many processing facilities already test for coliforms using coliform Petrifilm. Improving the ability of processors to detect PPC will improve the quality of the fluid milk supply.
\end{abstract}

Received October 25, 2019.

Accepted January 30, 2020.

*Corresponding author: nhw6@cornell.edu
Key words: Pseudomonas, fluid milk, postpasteurization contamination

\section{Short Communication}

Recontamination of fluid milk after pasteurization, or postpasteurization contamination (PPC), is a major hurdle to achieving high-quality fluid milk (Martin et al., 2018). Products that have PPC typically reach the Pasteurized Milk Ordinance (PMO) bacterial limit of $20,000 \mathrm{cfu} / \mathrm{mL}$ (FDA, 2017) within 7 to $10 \mathrm{~d}$ of pasteurization when stored at $6^{\circ} \mathrm{C}$ (Ranieri and Boor, 2009). Studies suggest that approximately $50 \%$ of fluid milk is contaminated with gram-negative bacteria (e.g., Pseudomonas), which is evidence of PPC (Alles et al., 2018; Reichler et al., 2018). This contamination often occurs sporadically at low levels and has frequently been associated with filling equipment (Martin et al., 2018). Further, the types of bacteria that are commonly reintroduced into fluid milk after pasteurization typically grow rapidly at refrigeration temperatures and can produce several enzymes that are deleterious to fluid milk quality, including proteases and lipases (Hayes et al., 2002). Indeed, fluid milk products with evidence of PPC have significantly lower sensory scores than samples showing no evidence of PPC (Reichler et al., 2018). Taken together, PPC is responsible for accelerated product quality deterioration and shorter shelf lives for fluid milk. To reduce the incidence of PPC, processors must detect the contamination quickly and reliably to eliminate the source of contamination.

For decades, the dairy industry has relied on coliform bacteria as indicator organisms for detecting unsanitary conditions and PPC (Martin et al., 2016). Coliforms are gram-negative, facultatively anaerobic, rod-shaped bacteria capable of fermenting lactose to produce gas and acid within $48 \mathrm{~h}$ at 32 to $35^{\circ} \mathrm{C}$ (Davidson et al., 2004) and are limited to $10 \mathrm{cfu} / \mathrm{mL}$ in grade A fluid milk by the PMO (FDA, 2017). Many methods have been developed to rapidly detect coliform bacteria in dairy products, yet contemporary studies of postpasteurization contaminants in fluid milk indicate that coliforms represent only a minor proportion of the 
total population of bacteria causing PPC (Alles et al., 2018; Reichler et al., 2018). Instead, studies indicate that Pseudomonas is the major organism responsible for PPC in contemporary fluid milk (Alles et al., 2018; Reichler et al., 2018). Pseudomonas is not a coliform and not a member of the Enterobacteriaceae (EB) family; therefore, it is not detected through the use of common methods used by the dairy industry to detect PPC (e.g., different methods that detect coliform and EB), and therefore such contamination may go undetected. Several studies have suggested the use of a medium that is capable of detecting all gram-negative bacteria, such as crystal violet tetrazolium agar (CVTA), as a better indicator of PPC in fluid milk (Van Tassell et al., 2012; Alles et al., 2018). Pseudomonas, coliform bacteria, and EB are all detected using CVTA.

Despite the demonstrated benefits of using CVTA for detection of PPC in fluid milk, industry adoption of this method has been slow. We hypothesize that this is due to (1) the lack of regulatory requirements for total gram-negative testing and (2) the fact that CVTA requires the use of traditional agar plates, which can be costly, labor intensive, and space inefficient. To address these issues, our goal for this study was to identify an alternative method to CVTA for detecting total gramnegative bacteria in fluid milk. Coliform Petrifilm (3M, St. Paul, MN), used frequently in the dairy industry to detect coliforms, is easy to use, saves space, and anecdotally has been known to allow for the growth of Pseudomonas and other non-coliform gram-negative bacteria after $48 \mathrm{~h}$ of incubation (personal communication, Kevin McGoldrick, 3M, St. Paul, MN). Previous studies have evaluated the ability of coliform Petrifilm and EB Petrifilm (3M) to enumerate select Pseudomonas species in experimentally contaminated fluid milk (Van Tassell et al., 2012); however, to our knowledge no published studies have evaluated the ability of these media to detect total gram-negative bacteria in naturally contaminated fluid milk products. Therefore, the goal was to evaluate the ability of coliform Petrifilm and EB Petrifilm to detect PPC in fluid milk compared with CVTA.

To that end, samples of fluid milk $(\mathrm{n}=33)$ were collected from 5 fluid milk processors in the northeastern United States in August 2018. Samples represented whole, $2 \%, 1 \%$, skim, and chocolate milk and were commercially packaged in various sizes (e.g., half gallon, quart, $8 \mathrm{oz}$ ). Samples were transported to the Milk Quality Improvement Program laboratory at Cornell University (Ithaca, NY) on ice and were aseptically distributed into individual sterile bottles for shelf-life testing at $6^{\circ} \mathrm{C}$ as previously described (Martin et al., 2012). Microbiological testing was performed on d 10, 14, 17, and 21 after pasteurization and included spiral plating on CVTA and plating undiluted fluid milk on both coliform Petrifilm and EB Petrifilm (3M) according to the manufacturer's instructions. All microbiological tests were conducted in duplicate. The CVTA plates were incubated at $21^{\circ} \mathrm{C}$ for $48 \mathrm{~h}$ (Frank and Yousef, 2004) before evaluation for the presence or absence of typical colonies (i.e., red colonies). Coliform and EB Petrifilm were incubated at $32^{\circ} \mathrm{C}$ and evaluated for the presence or absence of typical colonies (i.e., colonies with acid production and associated gas bubbles on coliform Petrifilm, and colonies with either acid production or associated gas bubbles on EB Petrifilm) as well as presence or absence of atypical growth at both 24 and $48 \mathrm{~h}$ of incubation.

Data were managed in Microsoft Excel (Professional Plus 2016; Microsoft Corp., Redmond, WA), and statistical analysis was performed in R (Kuhn, 2008). Microbiological testing data for the 33 samples were compiled and parsed such that the final data set included only sample results from a given shelf-life day (d 10, 14, 17, or 21) if all 8 tests were performed. Data were recorded as either negative (0) or positive (1) for detection of typical or any growth depending on the test. In total, 92 observations (1 per unique sample and shelf-life day) were retained, including 9 samples tested on 4 d, 8 on $3 \mathrm{~d}$, and 16 on $2 \mathrm{~d}$. Coliform and EB Petrifilm results from those 92 observations were compared with results from CVTA, and sensitivity, specificity, positive predictive value, negative predicative value, balanced accuracy, and accuracy $P$-value (i.e., accuracy of each test compared with the "no information rate," which is taken to be the largest class percentage in the data) were calculated using the caret package (Kuhn, 2008) in R. Data and code used for this study are available on GitHub (https://github.com/FSL-MQIP/Alternative -Gram-negative). Sensitivity describes the ability of each test to correctly identify a positive sample compared with the gold standard (i.e., CVTA), also known as the true-positive rate, whereas specificity describes the ability of each test to correctly identify a negative sample compared with the gold standard, also known as the true-negative rate.

Overall, of the 92 observations on the 33 samples evaluated here, 61 were positive for gram-negative bacteria on CVTA. Sensitivity, or true-positive rate, ranged from a low of 0.098 for both coliform and EB Petrifilm evaluated at $24 \mathrm{~h}$ for only typical growth to a high of 0.787 for coliform Petrifilm evaluated at 48 $\mathrm{h}$ for all growth (Table 1). Specificity, or true-negative rate, ranged from a low of 0.839 for coliform Petrifilm evaluated at $48 \mathrm{~h}$ for all growth to a high of 1.000 for coliform Petrifilm evaluated at 24 and $48 \mathrm{~h}$ for typical growth, EB Petrifilm evaluated at 24 and $48 \mathrm{~h}$ for typical growth, and EB Petrifilm evaluated at $24 \mathrm{~h}$ for 
all growth (Table 1). Taken together, the balanced accuracy, or the average between sensitivity and specificity, ranged from a low of 0.402 for both coliform and EB Petrifilm evaluated at $24 \mathrm{~h}$ for typical growth to a high of 0.804 for coliform Petrifilm evaluated at $48 \mathrm{~h}$ for all growth (Table 1). Coliform Petrifilm evaluated at $48 \mathrm{~h}$ for all growth was the only test combination that had a significant $P$-value $(P=0.002)$ for balanced accuracy out of the 8 tests evaluated (Table 1) compared with the "no information rate." It is unsurprising that coliform and EB Petrifilm evaluated for typical growth after $24 \mathrm{~h}$ of incubation resulted in the lowest balanced accuracy because these methods do not detect important PPC bacteria (namely Pseudomonas) that are detected using the reference method (CVTA). Importantly, 48-h coliform Petrifilm, which performed well, does allow for detection of Pseudomonas. For example, in a study by Alles et al. (2018), only $7 \%$ of fluid milk samples with evidence of PPC showed evidence of non-coliform EB and $20 \%$ of fluid milk samples with PPC showed evidence of contamination with coliform bacteria, whereas $100 \%$ of fluid milk samples with evidence of PPC showed evidence of contamination with non-coliform, non-EB bacteria (e.g., Pseudomonas). In another study, Reichler et al. (2018) found that in samples with spoilage due to PPC $(\mathrm{n}=132)$, Pseudomonas were the predominant causative organisms (found in 101 samples with evidence of PPC), whereas only 27 of the 132 samples with PPC showed evidence of coliform bacteria contamination.

Our study indicated that coliform Petrifilm evaluated at $48 \mathrm{~h}$ for all growth had the highest balanced accuracy (0.804) of all 8 test combinations compared with the current standard method for detecting total gram-negative bacteria, CVTA (Table 1). Anecdotally, coliform Petrifilm has been known to allow for breakthrough growth of non-coliform gram-negative bacteria after $48 \mathrm{~h}$ of incubation (personal communication, Kevin McGoldrick, 3M, St. Paul, MN). This was supported in our study by the improved sensitivity of coliform Petrifilm evaluated at $48 \mathrm{~h}$ for all growth (0.787) compared with coliform Petrifilm evaluated at $24 \mathrm{~h}$ for all growth (0.410). Importantly, even coliform Petrifilm evaluated at $24 \mathrm{~h}$ for all growth had a higher sensitivity than coliform Petrifilm evaluated at $24 \mathrm{~h}$ for typical growth (0.098), which is the current method used by the US dairy industry as an indicator of unsanitary conditions. Indeed, the coliform Petrifilm literature indicates that the growth of lactose non-fermenting bacteria after 24 $\mathrm{h}$ of incubation is typically due to non-coliform bacteria such as Pseudomonas, yet our data suggest that not all samples contaminated with Pseudomonas will be positive after $24 \mathrm{~h}$, necessitating the additional 24-h incubation period. Although CVTA has been shown

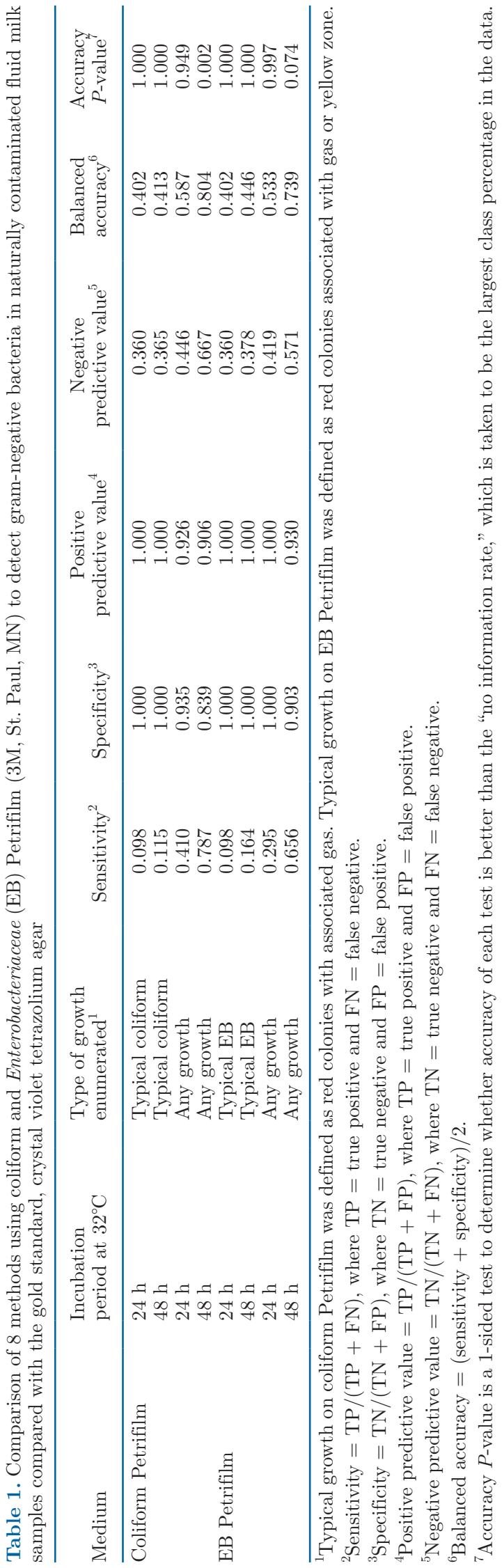


in multiple studies to outperform other methods for detection of Pseudomonas and other common fluid milk contaminants that cause PPC (Van Tassell et al., 2012; Alles et al., 2018), it is a traditional culture-based method that is inaccessible for many industry laboratories. Conversely, Petrifilms are used widely in the US dairy industry, specifically coliform Petrifilms. Leveraging this existing and common test, which is used to confirm that fluid milk is within the PMO limit of 10 $\mathrm{cfu} / \mathrm{mL}$ coliform, to test for a larger group of gramnegative contaminants including Pseudomonas, is a low-input way of improving the dairy industry's ability to detect PPC. It is notable that resolving PPC events often requires additional follow-up testing beyond the initial detection of gram-negative contaminants to identify the source of the contamination. We anticipate that the method used in our study will provide a useful tool for this follow-up testing but caution against using this method to specifically isolate Pseudomonas because there likely are variations in the individual bacterial strains capable of growing on coliform Petrifilm versus a non-selective medium or a medium specifically designed for total gram-negative bacteria.

Addressing PPC in fluid milk greatly depends on the ability of processors to detect contamination. Studies show that the primary bacterial organisms responsible for fluid milk PPC are not detected by coliform testing, the indicator group currently required by the PMO. To improve processors' ability to detect relevant bacterial contaminants in fluid milk, tests should be used that detect coliforms as well as Pseudomonas and other gram-negative bacteria. This study indicates that the coliform Petrifilm method, a test already in use by many processors, can be modified (i.e., incubation for $48 \mathrm{~h}$ and evaluation of all growth) to allow for growth of non-coliform gram-negative bacteria, improving the ability of coliform Petrifilm to detect total gramnegative bacteria compared with the standard method, CVTA. Although this method has reduced sensitivity and specificity relative to plating on CVTA, the ease of use and accessibility of coliform Petrifilm overall improves the ability of the dairy industry to detect PPC. Implementing this method, which is easily adoptable and uses pre-existing resources, will allow fluid milk processors to better respond to contamination events, leading to less PPC and improved fluid milk quality.

\section{ACKNOWLEDGMENTS}

This work was supported by the New York State Dairy Promotion Advisory Board (Albany, NY; OSP no. 88975), dairy farmers dedicated to the manufacture of high-quality dairy products. The authors acknowledge the students and staff of the Milk Quality Improvement Program and the Food Safety Laboratory at Cornell University (Ithaca, NY) who assisted with this project. The authors also acknowledge that Martin Wiedmann is a paid speaker for $3 \mathrm{M}$ (St. Paul, MN).

\section{REFERENCES}

Alles, A. A., M. Wiedmann, and N. H. Martin. 2018. Rapid detection and characterization of postpasteurization contaminants in pasteurized fluid milk. J. Dairy Sci. 101:7746-7756. https://doi.org/ 10.3168/jds.2017-14216.

Davidson, P., L. Roth, and S. Gambrel-Lenarz. 2004. Coliform and other indicator bacteria. Pages 187-226 in Standard Methods for the Examination of Dairy Products. 17th ed. H. M. Wehr and J. F. Frank, ed. American Public Health Association, Washington, DC.

Food and Drug Administration (FDA). 2017. Grade "A" Pasteurized Milk Ordinance. FDA, College Park, MD.

Frank, J. F., and A. E. Yousef. 2004. Tests for groups of microorganisms. Pages 227-248 in Standard Methods for the Examination of Dairy Products. 17th ed. H. M. Wehr and J. F. Frank, ed. American Public Health Association, Washington, DC.

Hayes, W., C. H. White, and M. A. Drake. 2002. Sensory aroma characteristics of milk spoilage by Pseudomonas species. J. Food Sci. 67:448-454. https://doi.org/10.1111/j.1365-2621.2002.tb11427.x.

Kuhn, M. 2008. Building predictive models in R using the caret package. J. Stat. Softw. 28:1-26. https://doi.org/10.18637/jss.v028.i05.

Martin, N. H., K. J. Boor, and M. Wiedmann. 2018. Symposium review: Effect of post-pasteurization contamination on fluid milk quality. J. Dairy Sci. 101:861-870. https://doi.org/10.3168/jds .2017-13339.

Martin, N. H., N. R. Carey, S. C. Murphy, M. Wiedmann, and K. J. Boor. 2012. A decade of improvement: New York State fluid milk quality. J. Dairy Sci. 95:7384-7390. https://doi.org/10.3168/jds .2012-5767.

Martin, N. H., A. Trmčić, T.-H. Hsieh, K. J. Boor, and M. Wiedmann. 2016. The evolving role of coliforms as indicators of unhygienic processing conditions in dairy foods. Front. Microbiol. 7:1549. https://doi.org/10.3389/fmicb.2016.01549.

Ranieri, M. L., and K. Boor. 2009. Short communication: Bacterial ecology of high-temperature, short-time pasteurized milk processed in the United States. J. Dairy Sci. 92:4833-4840. https:// doi.org/10.3168/jds.2009-2181.

Reichler, S. J., A. Trmčić, N. H. Martin, K. J. Boor, and M. Wiedmann. 2018. Pseudomonas fluorescens group bacterial strains are responsible for repeat and sporadic postpasteurization contamination and reduced fluid milk shelf life. J. Dairy Sci. 101:7780-7800. https://doi.org/10.3168/jds.2018-14438.

Van Tassell, J. A., N. H. Martin, S. C. Murphy, M. Wiedmann, K. J. Boor, and R. A. Ivy. 2012. Evaluation of various selective media for the detection of Pseudomonas species in pasteurized milk. J. Dairy Sci. 95:1568-1574. https://doi.org/10.3168/jds.2011-4958.

\section{ORCIDS}

S. I. Murphy (D) https://orcid.org/0000-0001-9092-0625 M. Wiedmann (D) https://orcid.org/0000-0002-4168-5662 N. H. Martin (1) https://orcid.org/0000-0003-1704-0634 